* This manuscript should be cited as:

Stevens, C. E., Jr., \& Zabelina, D. L. (in press). Classifying creativity: Applying machine learning techniques to divergent thinking EEG data. NeuroImage.

Classifying Creativity: Applying Machine Learning Techniques to Divergent Thinking EEG Data

Carl E. Stevens, Jr. \& Darya L. Zabelina

\author{
University of Arkansas \\ Department of Psychological Science \\ 480 Campus Drive
}

Fayetteville, AR 72701 USA

Correspondence concerning this article should be addressed to Carl E. Stevens, Jr., Department of Psychological Science, 480 Campus Drive, Fayetteville, AR 72701. Ph: 479-575-4256. E: cesteven@uark.edu. 


\begin{abstract}
Prior research has shown that greater EEG alpha power $(8-13 \mathrm{~Hz})$ is characteristic of more creative individuals, and more creative task conditions. The present study investigated the potential for machine learning to classify more and less creative brain states. Participants completed an Alternate Uses Task, in which they thought of Normal or Uncommon (more creative) uses for everyday objects (e.g., brick). We hypothesized that alpha power would be greater for Uncommon (vs. Common) uses, and that a machine learning (ML) approach would enable the reliable classification data from the two conditions. Further, we expected that ML would be successful at classifying more (vs. less) creative individuals. As expected, alpha power was significantly greater for the Uncommon than for the Normal condition. Using spectrally weighted common spatial patterns to extract EEG features, and quadratic discriminant analysis, we found that classification accuracy for the two conditions varied widely among individuals, with a mean of $63.9 \%$. For more vs. less creative individuals, $82.3 \%$ classification accuracy was attained. These findings indicate the potential for broader adoption of machine learning in creativity research.
\end{abstract}

Keywords: Alpha, Classification, EEG, Creativity, AUT 


\section{Classifying Creativity: Applying Machine Learning Techniques to Divergent Thinking EEG Data}

Barring a few early exceptions, (Martindale\& Mines, 1975; Martindale \& Hasenfus, 1978), the use of electroencephalography (EEG) has only recently become common in creativity research. The increased interest and utilization of EEG to assess creative cognition - the ability to generate novel and useful ideas (Sternberg \& Lubart, 1996) - is largely attributable to the EEG's excellent temporal resolution, which allows investigations of event-related potentials (ERPs), as well as transient network coherence and phase-locked activity. EEG-based research has been well-suited for highlighting the complex dynamics of creative cognition, bringing valuable insights to the field (for review, Stevens \& Zabelina, 2019).

\section{EEG and Divergent Thinking}

Divergent thinking (DT) tasks are a common and long-standing method for examining creative idea generation (Guilford, 1967). One standard way to assess DT is with an Alternate Uses Task (AUT). On the AUT, participants are asked to generate novel uses for common objects, such as a brick, and their responses are typically scored for fluency (i.e., number of uses generated) and originality (i.e., uniqueness of responses).

EEG-based work most consistently suggests that better performance on the AUT tasks is linked with increased alpha-band (8-13 Hz) activity (Benedek, 2018; Fink \& Benedek, 2014). Higher task-related power (TRP) in the alpha range, for example, has been found in the frontal and parietal regions when greater creative demands are imposed during the AUT (Jauk, Benedek, \& Neubauer, 2012). A positive association between alpha power and creativity has also been found in other tasks that have novel and normal response conditions (Zhou, Chen, Wang, Zhao, Zhou, \& Lu, 2018). Alpha power was reported to be primarily increased at the beginning of 
creative idea generation (when participants were asked to think of creative uses for common objects), dip, then increase again just prior to participants' responses (Schwab, Benedek, Papousek, Weiss, \& Fink, 2014). This U-shaped alpha effect was found to be particularly true of more creative individuals (Rominger et al., ,2019). Furthermore, alpha power was found to decrease over time in an open-ended task involving a change from more strongly divergent, to more strongly convergent, thinking (Zhou et al., 2018). When participants were asked to provide novel uses for the same object in four distinct, sequential response phases, both alpha and creativity ratings increased from the first to the later phases, possibly reflecting the greater creative demand involved in generating the additional responses (Agnoli, Zanon, Mastria, Avenanti, \& Corazza, in press).

Individual differences have also been reported at the trial level: More creative people have demonstrated higher alpha-band power than less creative individuals on the AUT (Fink \& Neubauer 2008). Additionally, those who perform better on the AUT have demonstrated greater duration of increased frontal and parietal alpha power (Camarda, et al., 2018), and show greater between-condition differences in frontal alpha TRP, compared to less creative individuals (Jauk et al., 2012).

The relationship between alpha-band power and creativity is thought to be at least partially attributable to reduced attention to external stimuli in favor of more internally oriented attention (Benedek, 2018). Specifically, participants might demonstrate greater creativity and alpha power on the AUT because they successfully inhibit information that is not relevant to the task at hand. Indeed, when attention is externally (vs. internally) oriented, alpha has been found to decrease (Benedek, Bergner, Könen, Fink, \& Neubauer, 2011). Visual processing can be 
demanding, and it is perhaps unsurprising that the simple act of closing one's eyes has been found to increase AUT performance (Ritter, Abbing, \& Van Schie, 2018).

Alpha, however, is not the only frequency band that has been shown to relate to successful AUT performance. Changes in delta- and theta-band activity have also been reported (Boot, Baas, Mühlfeld, de Dreu, \& van Gaal, 2017; Wokke, Ridderinkhof, \& Padding, 2018). Theta in particular appears to have an interesting relationship with DT, as evidenced by increased theta fronto-occipital functional connectivity in more (vs. less) creative individuals (Wokke et al., 2018). Previously, frontal theta was found to be associated with greater cognitive control (Cavanaugh \& Frank, 2014). Given the role of attention (internal vs. external) in DT (Benedek, 2018), this finding may point to the theta-dependent suppression of visual functions by frontal executive areas. In other words, theta activity may be an indication of top-down processing in the service of creative cognition. This view is supported by the implication of the lower end of the EEG spectrum (i.e., theta) in long-range communication between brain regions (Clayton, Yeung, \& Kadosh, 2015). In light of these findings, it is clear that the relationship between oscillatory neural activity and creativity is not confined to the alpha frequency band, though alpha's relationship to creativity is the most well-supported by current empirical investigations.

The practice of using machine learning (ML) techniques to understand the nature of EEG signals has grown substantially in recent years. In creativity research, however, this is a technique that is novel and ripe for implementation, with unexplored territory extending in every direction. The purpose of the present study was thus to move into this literature gap and investigate the extent to which more and less creative brain states can be reliably discerned according to their EEG characteristics, allowing for unbiased detection of creative thinking. 


\section{EEG and Machine Learning}

The growing use of machine learning in EEG research is at least partially attributable to the increased availability of code and software packages, as well as the ever-increasing computational capacity of modern computers, without the need to purchase a machine that can fill a small room. The purposes of studies of this nature have varied widely, and include attempts to detect disease (Orrù, Pettersson-Yeo, Marquand, Sartori, \& Mechelli, 2012), recognize emotional states (Giannakaki, Giannakakis, Farmaki, \& Sakkalis 2017), and even classify individuals by learning style (Jawed, Amin, Malik, \& Faye, 2019). In the case of brain-computer interface (BCI) applications, motor cortical patterns have been classified and used to control virtual and even physical objects (Abiri, Borhani, Sellers, Jiang, \& Zhao, 2018). This approach is of particular interest in fields of research such as stroke, paralysis, and other areas in which mobility is compromised.

ML is an attractive approach in the field of creativity research for several reasons. An important one is that, while certain EEG features that are known to be related to creativity can be specifically targeted in an ML paradigm (e.g., specific frequency band or channels), the "handsoff" nature of the algorithmic process, once it is set in motion, does afford researchers the possibility of discovering new features that are not expected. In other words, if the information that is used as ML input is broader in content than what would be assumed to be essential, a machine would be more likely to detect a previously unknown pattern. Further, while some features of the creative process are understood, based on the replication of results in studies from various laboratories (e.g., Benedek, Schickel, Jauk, Fink, \& Neubauer, 2014; Jauk et al., 2012; Schwab et al., 2014), it is still not obvious to assume that, at the individual level, everyone is creative in precisely the same way. In other words, people may utilize disparate strategies and 
resources to arrive at analogous creative ideas. Variability among individuals can cause difficulties for researchers when attempting to determine common functional or anatomical features among individuals. ML, however, makes it possible to detect EEG features that are subject-specific under certain experimental conditions, and make predictions about the nature of new information obtained from that same individual.

\section{Types and Procedures}

In order to classify EEG data, feature extraction is used to reduce data dimensionality and select the EEG features that are relevant for classification. Common algorithms include estimates of power spectral density (PSD), wavelet transform, and common spatial pattern (CSP) analysis. Once useful features are determined, a decision-making algorithm is used to classify the data. There are various types of algorithms, including the support vector machine (SVM), k-nearest neighbors (k-NN), artificial neural networks (ANN), and linear discriminant analysis (LDA) (Christensen \& Abdullah, 2018). Once the decision-making algorithm has made some determination regarding the nature of the EEG signal, this determination is output for the user.

The success of ML techniques in the classification of brain states can be assessed by accuracy during training, and by how accurately a trained model can categorize new data, if realtime feedback is desired. Training accuracy is typically assessed using a cross-validation procedure. Datasets are frequently divided into 10 parts, 9 of which are used for training, while the one remaining part is used to test the model's accuracy. This specific process is referred to as “10-fold cross-validation”. Validation outputs frequently include false-positive, false-negative, true-positive, and true-negative rates, and/or an overall accuracy rate.

Accuracy can vary considerably across studies, which is not surprising, given the diverse nature of the questions that are addressed in the literature, and the wide variety of tasks and 
analytical methodologies that are employed. There are many examples of ML's ability to classify mental state EEG data. For example, using an SVM on data from 12 bilateral pairs of electrodes, 4 different musically elicited emotions were correctly classified at an average rate of $82.29 \%$ across participants (Lin, Wang, Jung, Wu, Jeng, Duann, \& Chen, 2010). This was slightly better than the $81.52 \%$ accuracy they achieved using a multilayer perceptron. SVM, k-NN, and a leastsquares (LS) classifier were used to classify EEG responses to exciting and relaxing music, recorded from 27 pairs of bilateral electrodes (Duan, Wang, \& Lu, 2012). SVM performed best in this study, with an average rate of $80.08 \%$ accuracy across participants. It is noteworthy that classification accuracy can vary between individuals. In this study, for example, the lowest observed rate was $67.47 \%$, while the highest rate was $98.97 \%$.

It is possible to use very few EEG channels and achieve satisfactory results, as was demonstrated recently (Avinash, Dikshant, \& Seema, 2018). Using data from 3 bilateral pairs of electrodes and only from the theta band, LDA was $90 \%$ accurate in the classification of positive and negative responses to musical stimuli, while k-NN was $100 \%$ accurate when using 3 neighbors (k). The potential to classify whether participants liked or disliked music was investigated using a 14-channel EEG headset (Emotiv EPOC) and 4 different classification algorithms (SVM, k-NN, and 2 types of discriminant analyses; Hadjidimitriou \& Hadjileontiadis, 2012). The best-performing algorithm in this study was found to be k-NN, which achieved an accuracy rate of $86.52 \%$ using the complete dataset from all participants. Somewhat surprisingly, using only a single EEG channel (Pz-Oz) and a random-forest classifier, 5 sleep stages were correctly classified at a rate of $88.66 \%$ in healthy subjects, though accuracy dropped to $66.96 \%$ in a group with REM behavioral disorder (Boostani, Karimzadeh, \& Nami, 2017). 
ML/EEG has also been used with success in more strictly cognitive studies. In a schizophrenia study on working memory, task performance (correct or incorrect) was classified by SVM using five frequency components (high theta, low theta, alpha, beta, and gamma) taken from four different time periods (during baseline, encoding, retention, and retrieval stages) at three electrode sites (Fz, Cz, and Oz; Johannesen, Bi, Jiang, Kenney, \& Chi-Ming, 2016). The model successfully classified performance with $84 \%$ accuracy in healthy controls, with frontal gamma during the encoding stage and central theta during retention as the most heavily weighted features. In the same study, a second model was able to classify healthy controls and schizophrenia patients with $87 \%$ accuracy, with frontal theta during baseline and frontal alpha during retrieval as the most heavily weighted features. Cognitive load has also been predicted from EEG features that were extracted from data collected while participants completed the Raven's APM Set II, which uses 36 increasingly difficult manipulations of shapes to assess general intelligence (Friedman, Fekete, Gal, \& Shriki, 2019). The approach taken in this study involved extracting features from band power in delta, theta, alpha, beta, and gamma bands for each of 62 EEG channels, in addition to measures of neural complexity and connectivity. An XGB algorithm, which is decision tree-based, was used. Predictably, decreasing the number of categories by increasing the bin size of difficulty levels (fewer bins, more individual levels in each) increased prediction results. Interestingly, when considering all channels, the addition of complexity features did not improve results, while the addition of connectivity features did. Perhaps more interestingly, prediction quality improved when the number of channels included in the analysis was restricted between 12 and $30\left(r^{2}\right.$ peaks of 0.7 for 12 and 26 electrodes, in the case of spectral features only), and the inclusion of only 8 channels still yielded $95 \%$ of peak 
performance. In short, the best results were obtained when the number of bins and channels were limited.

To our knowledge, there is no empirical work in which DT was investigated using EEG/ML techniques. One study, however, reported in conference proceedings that moments of intense in-the-moment creativity (ITMC) were accurately discerned from less creative periods at a rate of $82.98 \%$ by an SVM algorithm (Carrol \& Latulipe, 2012). These ITMC moments were determined by external judges who watched participants sketch over the course of 30 minutes via video. Data analysis was restricted to the alpha and beta bands, and the limited-channel EPOC headset was used for acquisition. While this is only one study, when considered in combination with the broader field of EEG/ML research, it does point to the potential of similar methodologies in the pursuit of new perspectives on creative cognition. This potential is made more appealing by the results of a human-computer interface study, in which internal and external attentional states were classified using LDA (Putze, Scherer, \& Schultz, 2016). Researchers used a visual oddball task to heighten external attention, and several different tasks that require internal attention (mental math, word generation, and episodic memory). Classification accuracy using only band power features reached $80.4 \%$, and when ERP features were included, accuracy increased to $81.2 \%$. As previously mentioned, the direction in which one's attention is focused can be an indicator of changes in both creativity and alpha power, further demonstrating the potential for EEG/ML in the creativity field. Recognition of this potential was the primary motivation for the current study.

\section{Present Study}

The present study utilized a computerized version of an Alternate Uses Task (AUT) (adapted from Jauk et al., 2012). During the task, participants were presented with a common 
object, such as a "brick," and were asked to think of an "Uncommon", or unusual use for each object (experimental condition), or a "Normal", or common use for the object (control condition). Each trial consisted of four phases: 1) a reference phase, which presented participants with a "jittered" fixation cross; 2) a preparation phase, where participants saw a cue indicating the kind of use (normal or uncommon) that was required for the current trial; 3 ) an idea generation phase, where the name of the object was displayed until participants indicated they had thought of a response; and 4) a response phase, where participants typed their response.

Prior research on creativity has consistently found between-condition and betweensubject differences in the alpha range and beyond (for review, Stevens Jr. \& Zabelina, 2019). It was predicted that the use of ML would enable the reliable classification of multi-band EEG data from more and less creative task conditions, and from more and less creative individuals. In addition to the manipulation of various ML parameters, a priori-based channel selection (reduction) was explored for the purpose of mitigating computational strain.

\section{Methods}

\section{Participants}

Thirty healthy individuals participated in the present study ( 8 males, 21 females, mean age $=19.55, S D=1.99)$. One participant was excluded from all data analyses because the task instructions were not followed correctly, resulting in a final sample of 29 participants. Most participants self-identified as White/Caucasian (86.2\%). All participants had normal or corrected-to-normal vision. They were not diagnosed with any psychiatric disorders at the time and were fluent English speakers. They were not under the influence of excessive caffeine, unprescribed medication, or alcohol. The study was approved by the local institutional review 
board. All participants gave written informed consent prior to their sessions and were compensated with research credits.

\section{Experimental task and procedures}

The AUT was used as a measure of creative idea generation. In this task, participants were presented with 30 different common objects (see Appendix for a list of objects used in this study). Each object was presented twice - once in the Normal condition, and once in the Uncommon condition. Order of object presentation, and the order of condition for each object was computer-randomized for each session. Specifically, each of the 30 items was presented first in one of the two conditions, then all 30 were presented again, but in the opposite condition to their first appearance. In the Normal condition, participants were instructed to think of ordinary, common ways to use the objects. In the Uncommon condition, they were instructed to think of unusual, creative ways to use the objects. Participants were told they had up to 30 seconds to respond for each object, at which point the trial would time out. Participants were instructed to remain as still as possible, and limit eye movement and blinking except in between trials. All instructions were presented visually, on a computer screen, and verbally, by a researcher.

Each of the 60 trials in the AUT began with the presentation of a fixation cross for a duration that was randomized between 2 and 5 seconds in 25 millisecond increments $(\sim 3.5 \mathrm{~s})$. Next, a cue word, either "Normal" or "Uncommon", was displayed for 5 seconds. This was followed by the name of an object, which remained on the screen for up to 30 seconds. To respond, participants hit the 'Enter' key on the computer keyboard. This interrupted the object presentation and brought up a white text box in which to type. Participants typed their responses, then hit 'Enter' again to record the answer and move on to the next trial (Figure 1). Participants were told it was okay to move their eyes freely while typing, but to keep them still again once the 
next fixation cross appeared. Trials were presented in 4 blocks of 15 trials, with a user-defined break period between blocks. The task typically took about 35 minutes to complete.

Fixation

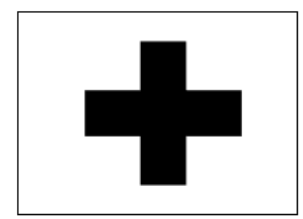

N3.5s
Preparation

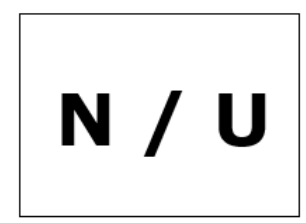

Generation

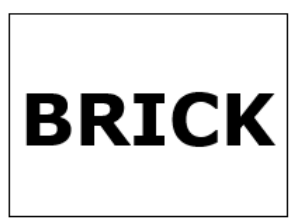

Response

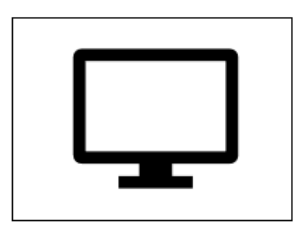

untimed

30s max

5s

Figure 1. Alternate Uses Task (AUT) phases and timing.

The task was custom coded using Presentation ${ }^{\circledR}$ software (Version 20.2,

Neurobehavioral Systems, Inc., Berkeley, CA, www.neurobs.com). Port codes appearing in the EEG time series files were programmed to identify the occurrence of experimental elements. These included unique codes to distinguish the presentation of Normal and Uncommon condition cue words and object names. This allowed models to be trained to classify epochs during which 2 different kinds of thinking occurred.

In order to examine individual differences in creativity, and conduct score-based creativity classification analysis, 5 independent judges blind to the conditions and the purpose of the study (all undergraduate psychology students, 1 male, 4 female, $M$ age $=20.2, S D=1.17$ ) rated all responses for creativity ("How creative is this response?"). Ratings were made on a Likert-type scale from 1 (least creative) to 10 (most creative). Raters achieved good inter-rater reliability (Cronbach's $\alpha \mathrm{s}>.80$ ). Two groups (High- and Low-creative) were formed by selecting the top and bottom 9 participants (middle excluded), based on the ratings. 


\section{EEG recording and equipment}

EEG signals were acquired using the Biosemi ActiveTwo system, with 32 scalp and 4 EOG AG/AgCl electrodes positioned according to the 10/20 system. Linked mastoid electrodes were used for reference. EEG data were recorded at $2048 \mathrm{~Hz}$ using the BioSemi ActiveTwo biopotential measurement system (BioSemi, Amsterdam, The Netherlands) and ActiView software (BioSemi). During recording, signals were high-pass filtered at $0.16 \mathrm{~Hz}$. Recordings were monitored continuously during experimental sessions, and all potentially artifact-producing events (coughing, sneezing, obvious muscle-based or other noise) were recorded by a researcher. No subjects had out-of-the-ordinary artifact-producing issues (sneezing or coughing spells, etc.). Both raw and cleaned data (process described below) were used separately in analyses (i.e., transitory events like blinks, muscle twitches, etc, were not removed prior to raw data feature extraction and classification). This was done with the intent of determining the accuracy that could be attained with minimal processing.

\section{General Analysis Strategy}

ML classification analyses of condition types (Normal and Uncommon) and creative types (High and Low) were the focus of this study. Between-condition spectral analyses focusing on the 8-30 Hz frequency range were also performed using cleaned data (see Spectral Analyses section for details). This was done to provide insight into the effectiveness of the task design to elicit condition-related changes in brain activity. Classification was performed on EEG data in two forms - raw and cleaned. This was done to allow a comparison of ML results derived through a normal offline EEG processing pipeline, and those that might be obtained through realtime processing. In both cases, variations in the following aspects of EEG data selection were examined: electrode locations/channels, time windows, frequency ranges (see Machine Learning 
Analyses section). The variations that were tested were predetermined, and those that are reported here were chosen based on classification performance; although, reporting on the 1-2.5 second window that comprises most results was predetermined (see Machine Learning Analyses section). Additional analyses were performed to assess the possible influence of artifacts on classification (see A Smaller, Earlier Analysis Time Window section, and Assessing the Potential Impact of Artifacts on Classification section).

\section{Data processing}

All signal processing occurred in MATLAB R2018b (The MathWorks, Inc., Natick, Massachusetts, United States) using the EEGLAB (version 14.2.1; Delorme and Makeig, 2004) and BCILAB toolboxes (developer version; https://github.com/sccn/BCILAB) (Kothe \& Makeig, 2013), which are made freely available by the Schwartz Center for Computational Neuroscience. Additional statistical analyses were carried out using IBM SPSS Statistics for Windows, version 25.

\section{Spectral analyses}

EEG signals were high-pass filtered at $1 \mathrm{~Hz}$ and low-pass filtered at $58 \mathrm{~Hz}$. Fifty-eight $\mathrm{Hz}$ was above the frequency range of interest, based on the literature, and setting the cutoff at this point filtered out frequencies in the range of line noise. Channel locations were assigned, and signals were referenced to the averaged mastoids (M1 and M2). Artifact Subspace Reconstruction (developed by Christian Kothe as part of BCILAB's online cleaning, now available for offline use) was used to identify and reject artifactual principle components. ASR uses a sliding window ( 0.5 seconds, $50 \%$ overlap) to remove transient, high-amplitude artifacts (e.g., eyeblinks, muscle twitches, etc.), after which rejected component activity is reconstructed from surviving components. Infomax ICA (Bell \& Sejnowski, 1995) was performed. Multiple 
Artifact Rejection Algorithm (MARA) (Winkler, Brandl, Horn, Waldburger, Allefeld, \& Tangermann., 2014; Gabard-Durnam, Mendez Leal, Wilkinson, \& Levin, 2018), which extracts 6 features from the temporal (1. mean local skewness), spatial (2. range within pattern, 3. current density norm), and spectral (4. log alpha power, 5. lambda, and 6. fit error) domains, was used to identify and remove "bad" independent components (ICs). MARA's default component rejection threshold of $50 \%$ probability - that a component is artifactual - was altered in MATLAB to a more conservative $80 \%$. Statistical comparisons were carried out using EEGLAB.

\section{Machine learning analyses}

Unfiltered EEG data (raw .bdf files), as well as datasets that were processed as noted in the previous section (cleaned data), were loaded in BCILAB. The continuous data were analyzed at both the individual and group levels. Data epochs of interest were selected in BCILAB using EEGLAB functions (BCILAB is designed primarily for real-time processing and will not process non-continuous, pre-epoched data as the program stands). These were defined as the periods from 1 to 2.5 seconds after stimulus onset, i.e., the appearances of object names. Additional latencies during the AUT task were also tested: an earlier, shorter interval immediately following the beginning of creative ideation ( 0.75 to 1.75 seconds); from -4 to -1 seconds, in relation to the onset of ideation (during the 5-second Normal or Uncommon preparation phase); and from -2 to -0.5 seconds, in relation to response (button press). These other intervals, however, yielded inferior classification accuracy. Additionally, prior to testing of these other intervals, the 1-2.5 second time period was selected based on a previous AUT study (Schwab et al., 2014), in which it was found that alpha band task-related power changes at dorsal frontal (F3/F4) and parietal (P3/P4) sites were greatest in the first of three evenly divided intervals beginning at the onset of idea generation (from 1-9 seconds post-onset, 2.67 seconds per interval), with no difference in 
TRP between hemispheres. Port codes sent from Presentation created event markers in the datasets to indicate these timepoints, as well as the occurrences of other task events. The 2 condition types (Normal and Uncommon) were distinctly identified by these markers.

Channel combinations of different quantities and from different locations on the scalp were systematically tested in order to find the best possible classification accuracy. These included a full-scalp combination (all 32 cortical channels), and P4 alone. In all cases, combinations included P4 (right parietal), given the consensus among studies regarding this region's involvement in creative ideation (Benedek, 2018). The locations of other individual channels that were combined with P4 were Fz, F3, F7, Cz, C3, Pz, P3, P7, T7 and the matching contralateral channels of each non-midline (z) channel. Channel combinations that were combined with P4 were as follows: (2 additional channels) F3/F7 (left frontal), F3/C3 (left frontocentral), C3/P3 (left centroparietal) C3/T7 (left centrotemporal), and the matching contralateral pairs of each; (3 additional channels) C3/C4/P3 (bilateral centroparietal), and P3/P7/P8 (bilateral parietal); (4 additional channels) F3/F4/F7/F8 (bilateral frontal), F3/F4/C3/C4 (bilateral frontocentral). These channel combinations were chosen to provide good coverage of cortical regions that have either been observed to be differentially responsive to the demands of the Normal and Uncommon AUT conditions (Jauk et al., 2012), or responsive in the early stages of creative ideation (Rominger et al., 2019; Schwab et al., 2014). The primary motivation for attempting classification using mainly smaller channel groups was twofold: 1) to reduce computational expense, and 2) to potentially enhance the performance of the feature extraction and classification algorithms by not including data that were unlikely to be helpful for classification. 
EEG features were extracted using 3 iterations of a Spectrally Weighted Common Spatial Patterns (SpecCSP) algorithm (Ramoser, Muller-Gerking, \& Pfurtscheller, 2000). Initial frequency bands of interest were theta $(4-8 \mathrm{~Hz})$, low alpha $(8-10 \mathrm{~Hz})$, high alpha $(10-13 \mathrm{~Hz})$, beta $(13-30 \mathrm{~Hz})$, and gamma $(30-42 \mathrm{~Hz})$, though theta and gamma were later excluded to enhance classification performance. Results therefore are restricted to data from the low-alpha, high-alpha, and beta bands. The minimum-phase filter frequency specification was set at $6 \mathrm{~Hz}$ at the low end, and $44 \mathrm{~Hz}$ at the high end. The number of SpecCSP patterns was set to no greater than the number of channels selected for a given dataset, i.e., 2 channels $=2$ CSP patterns (errors occur otherwise). To reduce computational load, data were downsampled to $100 \mathrm{~Hz}$ at this stage, in accordance with the Nyquist Theorem (digital sample rate must be $>=2 \mathrm{x}$ the highest analog frequency under analysis).

The types of supervised classification algorithms used were quadratic discriminant analysis (QDA) and Support Vector Machine (SVM). QDA differs from LDA in that it can learn quadratic boundaries, as opposed to linear boundaries, and is therefore more flexible. This is important when data classes are not linearly separable. Also, in contrast to LDA, QDA does not require that different classes have identical covariance matrices (Bhattacharyya, Khasnobish, Konar, Tibarewala, \& Naga, 2011). The quadratic discriminant function is $\delta k(x)=-1 / 2 \log \left|\Sigma_{k}\right|-$ $1 / 2\left(x-\mu_{k}\right)^{T} \sum^{-1}{ }_{k}\left(x-\mu_{k}\right)+\log \pi_{k}$. SVMs are discriminative classifiers that are very commonly used in neurophysiological data classification. The purpose of this algorithm is to find a linearly separable hyperplane, or decision boundary, by which to differentiate classes (Rossi \& Villa, 2007). The margin between the plane and the nearest data points on either side, which are known as support vectors, is maximized. If a decision boundary cannot be found, SVM will search after transforming the data to a higher dimension. Because of its ubiquity in biosignal classification 
analyses, it was deemed important to include SVM in testing on the current dataset. As noted in the results section, however, QDA consistently outperformed SVM in the current study, so the reported results are restricted to QDA.

For both classifier types, predictive models were trained on SpecCSP-derived features using a leave-one-out 10 -fold cross-validation performance estimation procedure ( 1 section held out for testing, 9 sections used for training, repeated for each section; 5 trials around any test trial excluded from the training set). During the task, distinct event codes marking the onset of Normal and Uncommon ideation periods were added to the data stream by Presentation. These were used as target markers (classifiers). For the classification of High- and Low-creative participants, in-house code was used to modify Uncommon condition event codes for the Highcreative group. This differentiated the intervals of interest for the High- and Low-creative groups from one another. Performance estimates at the group and individual levels are described in terms of classification accuracy, as determined by true/false positive and negative rates.

\section{Results}

Participants responded faster in the Normal condition $(M=1.99 \mathrm{~s}, S D=0.83)$ than in the Uncommon condition $(M=9.23 \mathrm{~s}, S D=2.58), t(28)=16.95, p<0.001, d=3.14$. This result was not surprising, given that the Normal responses were obvious in nature, and were expected to be easier to generate.

Paired $t$-tests were used to examine between-condition log-transformed power spectral density (PSD) in EEGLAB. All subjects' cleaned data were included. For the Uncommon condition, power was significantly greater than in the Normal condition in a number of frequency ranges at F4 (Figure 2). This included a continuous range spanning the low- and highalpha bands $(8.6-10 \mathrm{~Hz}$ and $10-11.5 \mathrm{~Hz}), t(28)=2.35, p=0.013$, and $t(28)=1.96, p=0.03$, 
respectively. Power was also greater in the Uncommon (vs. Normal) condition in numerous ranges of the beta band between 17.5 and $27.6 \mathrm{~Hz}(p s<0.05)$. Power was also greater at $\mathrm{P} 4$ for the Uncommon (vs. Normal) condition, including much of the low- $(8.6-10 \mathrm{~Hz})$ and high-alpha bands $(10.3-11.8 \mathrm{~Hz}), t(28)=1.75, p=0.04$, and $t(28)=1.74, p=0.05$, respectively). Much like $\mathrm{F} 4$, though over a greater portion of the frequency band, power at P4 was greater in numerous ranges of the beta band between 12.9 and $20.1 \mathrm{~Hz}$, and at $28 \mathrm{~Hz}(p s<0.05)$ (Figure 2).
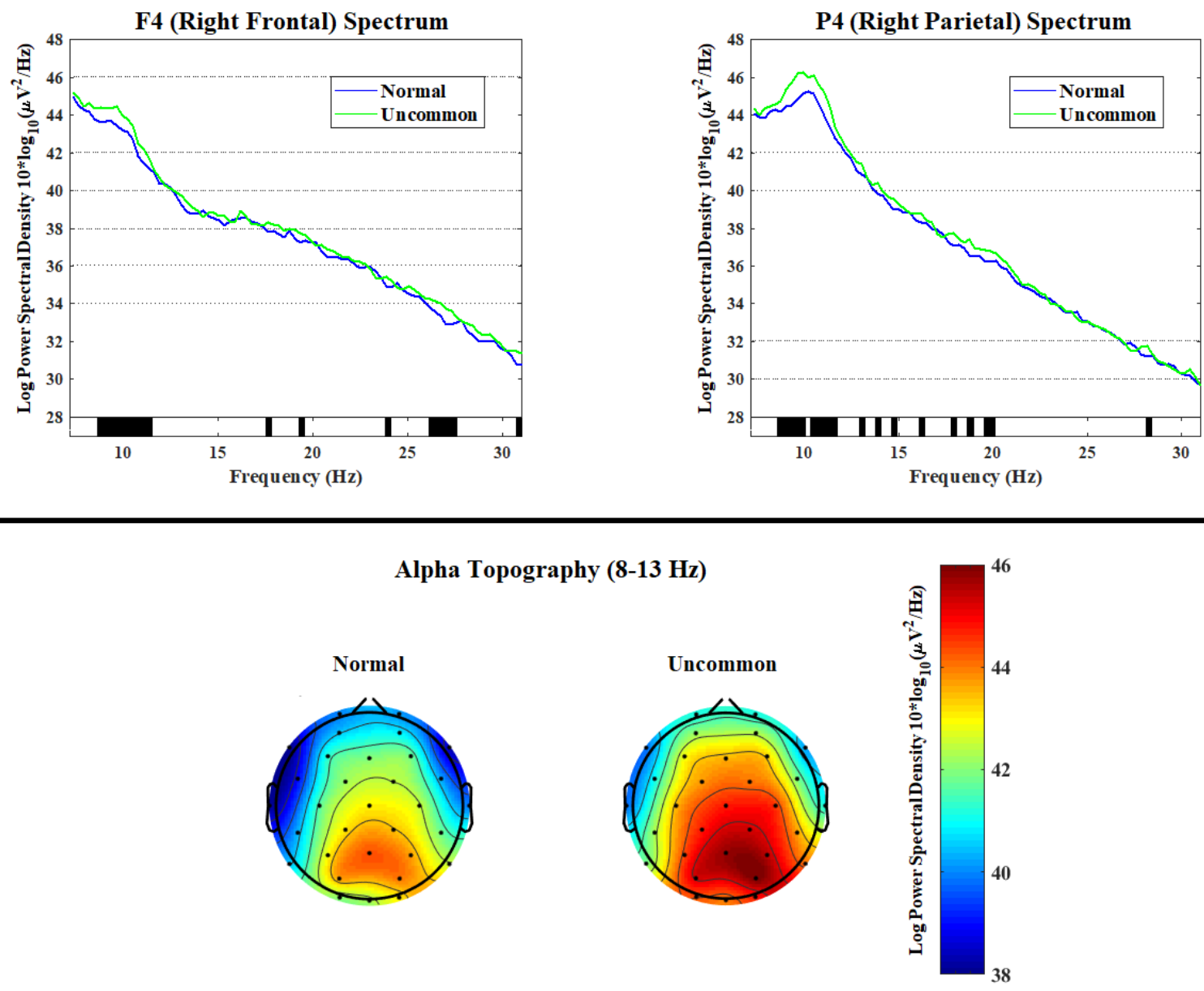

Figure 2. Between-condition (Normal versus Uncommon) comparison of spectral power density at F4 and P4 electrode sites. Black bars along the $\mathrm{x}$-axis indicate frequencies where differences between conditions are significant at the level of $p<0.05$. The topographic map (all channels) at bottom shows power at $10 \mathrm{~Hz}$ (alpha) for the two conditions separately. 
Though the difference was typically minor, QDA classification in BCILAB outperformed that of SVM in all cases, so QDA results are reported. Classification accuracy generally maintained an inverse relationship with the number of channels that were included. Accuracy was indeed found to be highest when restricting the number of channels to right frontal (F4) and right parietal (P4). In addition to a reduced number of features and increased accuracy, this restriction provided the benefit of reduced computational expense. The EEG classification results reported below are therefore restricted to the F4/P4 channel combination.

\section{Condition Classification Results: Individual Level, All Participants}

Ten-fold cross-validation of QDA condition classification for individual participants was assessed using both raw signals and cleaned data separately. For raw data, individual accuracy rates ranged from $36.7 \%$ to $93.3 \%$, with a mean of $63.9 \%(S D=0.15)$. For cleaned data, individual accuracy ranged from $39 \%$ to $95 \%$ with a mean of $62.7 \%(S D=0.15)$.

\section{Condition Classification Results: Whole-Group Level}

For whole-group condition classification, all participants' data were concatenated and used for model training. Raw and cleaned data were used separately to train and evaluate models. Score-based (AUT task ratings) classification of more and less creative individuals was also performed using both raw and cleaned data from the Uncommon condition only, thus minimizing the impact of fast responses, which were common in the Normal condition. Due to the extremes in classification accuracy among individuals, whole-group classification accuracy was generally poorer than individual classification.

Using all participants' raw data, the condition classification accuracy for QDA was 59.7\% (Figure 3). Additionally, when a QDA-derived model was trained on all participants' raw 
data, and the model was then applied to individuals, classification accuracy ranged from $40 \%$ to $85 \%$, with a mean of $59.2 \%(S D=0.12)$.

\section{Classification of High- and Low-creative Individuals}
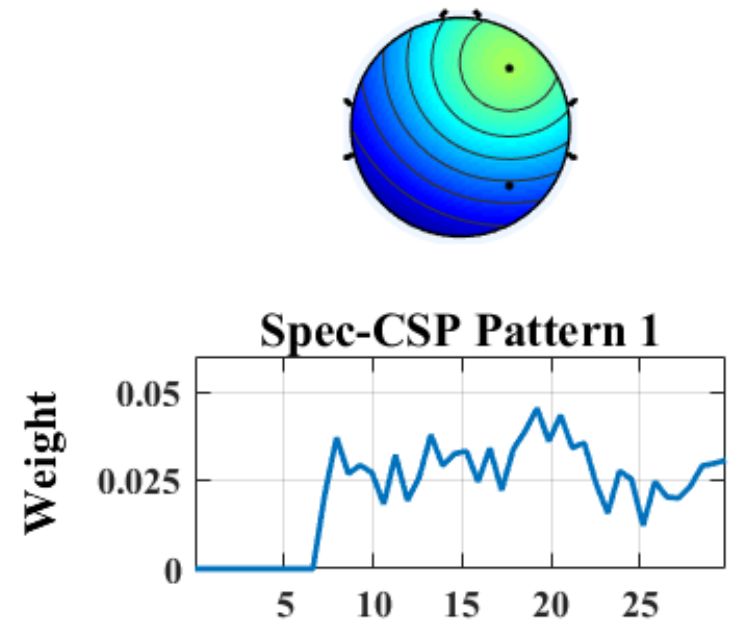
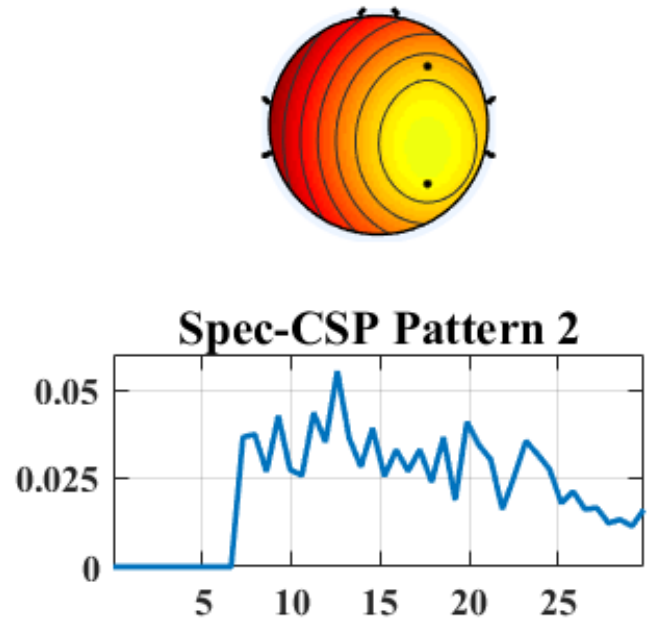

Frequency in $\mathrm{Hz}$

\section{Condition Classification}
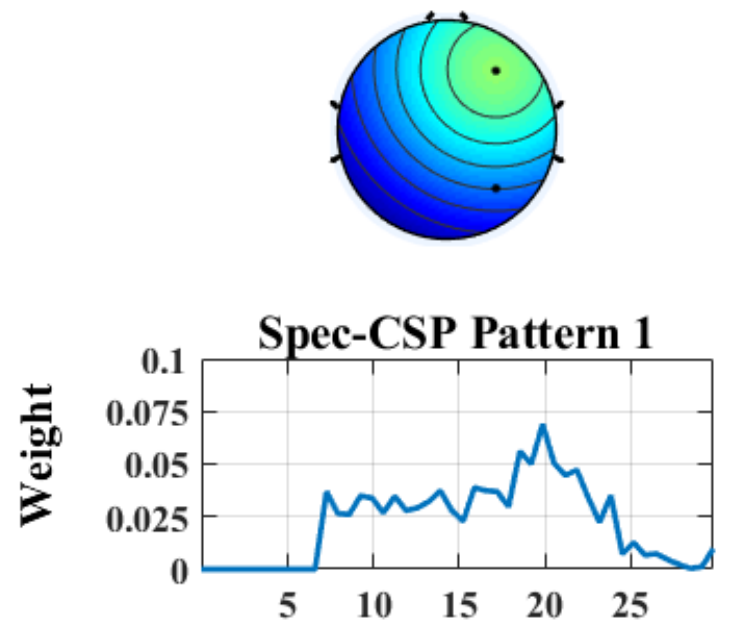
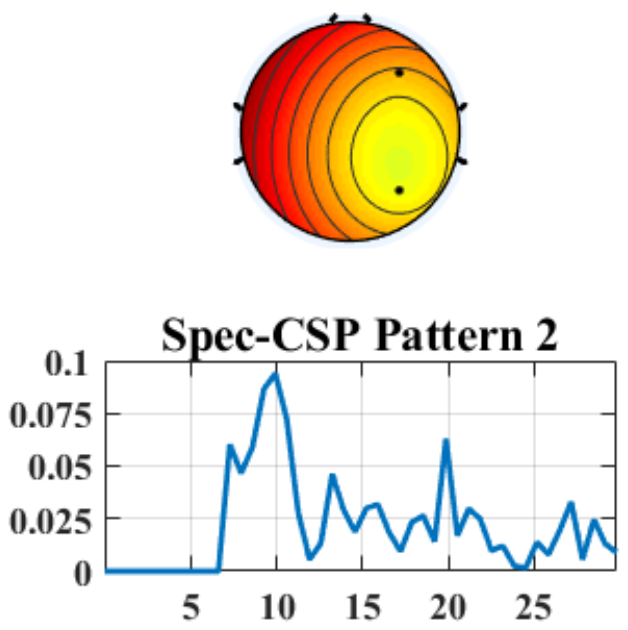

Frequency in $\mathrm{Hz}$

Figure 3. Spectrally weighted common spatial patterns (Spec-CSP) found in High- and Lowcreative groups (top), and in whole-group condition classification analyses (bottom). Two EEG channels (black dots) were used for classification: F4 (right frontal) and P4 (right parietal). Spectral patterns are shown beneath the topographic maps. Relative to the rest of the spectrum, frontal patterns (upper and lower left side) exhibit heavily weighted beta (13-30 Hz), and parietal patterns (upper and lower right side) exhibit heavily weighted alpha $(8-13 \mathrm{~Hz})$. 
Accuracy using all participants' cleaned data was slightly lower than for raw data.

Condition classification accuracy for QDA was 57.5\% (+/-11.8\%; folds $=10)$, and when a QDAderived model that was trained on all participants' cleaned data was applied to each individual, predictive accuracy ranged from $47.4 \%$ to $88.3 \%$, with a mean of $58.5 \%(S D=0.13)$.

\section{Condition Classification Results: High- and Low-Creative Groups Separately}

For raw data classification, High-creative group accuracy was 56.3\% $(+/-11.7 \%$; folds $=$ 10). Increasing the number of folds decreased accuracy slightly, to $56.1 \%(+/-13 \%$; folds $=25)$. Low-creative group accuracy was slightly better, at 58.1\% $(+/-11.2 \%$; folds $=10)$, and 59.2\% $(+/-13.7 \%$; folds $=25)$.

QDA condition classification accuracy was $54.9 \%$ for cleaned High-creative group data $(n=9)$. Increasing the number of folds increased accuracy to $56 \%(+/-14.8 \%$; folds $=25)$.

Condition classification accuracy for cleaned Low-creative group data $(n=9)$ was slightly better, at $56.9 \%(+/-13.4 \%$; folds $=10)$. Increasing the number of folds increased accuracy to $58.5 \%$ $(+/-14.9 \%$; folds $=25)$.

\section{Group Classification Results: High- and Low-Creatives}

For raw data, between-group creativity classification was $82.3 \%(+/-12.7 \%$; folds $=10)$. For 5 folds, accuracy was $80.6 \%(+/-11.3 \%)$. As was the case for cleaned data, raising the number of folds to 25 reduced accuracy, compared to using $10(81.2 \%,+/-15.7 \%)$.

For cleaned data, classification of the High-and Low-creative groups) was $81.3 \%(+/-$ $13.2 \%$; folds $=10)($ Figure 3$)$. At only 5 folds, accuracy was $79.6 \%(+/-10.7 \%)$. Raising the number of folds to 25 did not improve accuracy, but rather reduced it, relative to using 10 folds $(80.7 \%,+/-16.4 \%)$ 


\section{A Smaller, Earlier Analysis Time Window}

There was initial concern on the part of researchers that a time window starting at 0.5 seconds could include stimulus response features that would be non-specific to either condition, thereby hampering classification. This early period was therefore not included in the original analysis plan. This thinking is somewhat in line with the earliest analysis window of 1-3.67 seconds used by Schwab et al. (2014). Because of the typically fast reaction times observed in the Normal condition in this study, however, a window from 0.5 to 1.75 seconds was added to the analyses. This avoided the mean Normal condition RT of 1.99 seconds, though some responses are still unavoidably present in this window. For condition classification, accuracy was lower in the $0.5-1.75 \mathrm{~s}$ window than in the $1-2.5 \mathrm{~s}$ window that has been described for raw data ($5.5 \%)$ and for cleaned data (-5.69\%). For creativity-based classification, accuracy was higher in the $0.5-1.75 \mathrm{~s}$ window than in the $1-2.5 \mathrm{~s}$ window for raw data $(+5.8 \%)$ and for cleaned data $(+3.1 \%)$

\section{Assessing the Potential Impact of Artifacts on Classification}

Due to RT-related concerns that response artifacts might have influenced classification using the 1-2.5s window, additional analyses were conducted to assess their possible influence. For all subjects, the mean number of Normal epochs (30 total) containing responses was 23.66, $S D=5.81$. Uncommon trials (also 30) contained 4.62 responses on average, $S D=5.08$. This between-condition difference was significant, $t(28)=13.3, p<0.01$. Response-containing epochs for High-creative $(M=18.89, S D=7.36)$ and Low-creative $(M=19, S D=8.29)$ groups were also assessed. When the between-condition difference scores for each group were compared, no between-group difference was found, $t(16)=-0.03, p=0.49$. 
Further testing of EEG data for the presence of artifacts was conducted. Using automated thresholding, cleaned data were tested for abnormal voltage values (+/- $75 \mu \mathrm{v})$ and abnormal spectra (for eye movements, $+/-50 \mathrm{~dB}$ in the $0-2 \mathrm{~Hz}$ window; for muscle, +25 or $-100 \mathrm{~dB}$ in the 20-40 Hz window) in EEGLAB. For all subjects, the number of trials that were exceeded these thresholds in the Normal condition was $32(M=1.1, S D=3.55)$; in the Uncommon condition, 45 trials exceeded thresholds $(M=1.55, S D=4.52)$. There was not a significant between-condition difference, $t(28)=-1.61, p=0.12$. A further test of between-group (High/Low creativity) differences in the number of threshold-exceeding trials also revealed no significant difference, $t(16)=0.61, p=0.55$. Visual inspection of cleaned data also did not reveal typical blink or lateral eye movement artifacts.

\section{Discussion}

The present study utilized an Alternate Uses Task (AUT) on which participants were presented with a common object and were asked to either think of Uncommon (creative) or Normal (not creative) ways to use these objects. Participants' responses were judged by independent raters for creativity, and two groups (High- and Low-creative) were formed based on their responses. Prior research found EEG differences between more and less creative conditions, and between more and less creative individuals (Benedek, 2018), thus we predicted that a machine learning approach would be able to classify the two task conditions, and the two groups.

\section{Classification of Uncommon and Normal Responses}

Overall, as predicted, the study was successful at classifying the creative states, as well as creative groups. EEG Spectral comparisons revealed the expected between-condition differences for the group as a whole. Specifically, right frontal and parietal alpha-band power was greater in 
the Uncommon, compared to the Normal condition at the beginning of creative ideation. Other studies have observed greater alpha TRP in Uncommon, compared to Normal conditions (Jauk et al., 2012), and increased alpha TRP during creative ideation (Benedek et al., 2014), highlighting the importance of alpha in creativity. Response times in the Uncommon condition were significantly slower than in the Normal condition. This finding is similar to those of other studies (Jauk et al., 2012), and provides external validity that participants were more likely to provide responses that came to mind quickly in the Normal condition. In the Uncommon condition, participants took longer, likely because they rejected obvious, typical ideas about object use in favor of less obvious, and thus more creative, ideas.

The most relevant channel combination for accurate classification was right frontal and parietal electrodes, F4 and P4. This restricted selection was beneficial not only in terms of classification accuracy, but it also reduced computational expense. This finding bodes especially well for future studies involving real-time data processing and neurofeedback.

In addition to the main period of 1-2.5 seconds that is reported in results, the general analysis strategy (subsection in Methods) included testing of several other latencies during the AUT task. These periods included the $0.75-1.75$ second post-stimulus window, a period during the preparation phase, in which participants were shown the cue word indicating which type of thinking they were about to do (Normal or Uncommon), and in the period just before responding. No other time period, however, yielded the accuracy found by selecting the period just after the name of each object appeared, barring the initial period to process the stimulus. Indeed, numerous periods just after stimulus onset were also tested, and the period reported here (1-2.5 seconds) yielded the greatest classification accuracy. This finding is corroborated by the results of a previous study where alpha power increased at the beginning of the creative ideation phase 
of a trial, then decreased (Rominger et al., 2019; Schwab et al., 2014). Although this previous work also found alpha increases just prior to responding, classification of data from this period was not as successful in the current study as data from the beginning of the creative ideation period.

In terms of classification accuracy, individual models generally worked better than group models, which is expected, given the individual difference variability in processing styles, task focus, and other variables. Participants whose models achieved the highest accuracy rates also demonstrated greater power in the alpha and beta bands at both electrode sites (F4/P4) than those whose models were less accurate. The most notable differences between High- and Lowaccuracy groups at right frontal and parietal regions resided in both the low- and high-alpha ranges - primarily 9-12 Hz - though power was generally greater for the High-accuracy group from about $25 \mathrm{~Hz}$ down to the low end of the frequency spectrum. The reason for this difference is somewhat unclear, though a trend was found for the link between higher creativity scores and increased classification accuracy. This leaves some ambiguity as to the reason(s) for the observed differences between High- and Low-accuracy groups, though the decreased signal power across much of the analyzed spectrum $(8-30 \mathrm{~Hz})$ introduces the possibility of a difference in overall signal quality for Low-accuracy data.

\section{Classification of More and Less Creative Individuals}

Prior research has pointed to the differences in the EEG characteristics of more, compared to less creative people (Beaty, Seli, \& Schacter, 2019; Benedek, 2018). In the current study, more versus less creative participants were successfully classified at a rate of just over $82 \%$, in the case of raw data. This level of between-group classification accuracy is encouraging, in terms of continued research in this vein. Specifically, there is the possibility of using models 
derived from highly creative individuals to provide real-time feedback to other participants in a training paradigm. Given the outperformance of individualized classification, however, expectations of this nature should be temperate. It is possible that a model derived from highly creative individuals will simply not provide helpful feedback to those whose EEG features match it poorly. A more likely benefit of continued research in this area is a richer understanding of creative thinking through the use of data-driven, ML analyses.

To our knowledge, this is the first ML-based EEG study on divergent thinking, thus variations in AUT parameters, the use of different types of DT tasks, and the adoption of everemerging and improving ML methods are all potential candidates to be used in this pursuit. Lastly, the observation that the classification of raw data resulted in accuracy rates that were essentially equivalent to the rates achieved using cleaned data highlights the possibility of successfully classifying EEG data in real time without the need for ultra-high-performance equipment. In other words, if computationally expensive data processing, like ICA, is not necessary to achieve good results, then research of this kind is not restricted to labs that possess machines that can perform such computations at break-neck speeds.

\section{Limitations}

One potential limitation is unbalanced male/female ration ( 8 males, 21 females). While it is possible that sex differences in EEG patterns could be an issue for classification in some circumstances, it is unclear that such is the case in this study. It was recently found that a deep learning network was able to classify males and females with $80 \%$ accuracy (Putten, Olbrich, \& Arns, 2018), however, researchers used a deep convolutional neural network applied to raw EEG. Thus, their analysis method differed substantially from that of the current study, so it is not possible to infer from their results that the current approach would be sensitive to sex 
differences. As a precaution, a 2-tailed t-test comparing male and female classification accuracy was performed, but no no sex differences were found.

Another limitation is the use of a 1-2.5 second time period used for classification, given the 1.99-second mean response time in the Normal condition. This complication was not anticipated. Given the self-paced task design, it raised the possibility that response-related factors might have played a role in the observed accuracy rates. The additional analysis of an earlier interval (0. 5-1.75 seconds) that did not contain the mean response time, however, yielded higher accuracy rates, despite prior concerns that features related to stimulus onset, not condition type, could muddy the waters for classification. Additional analyses also revealed that while there was a highly significant between-condition difference in the number of Normal and Uncommon trials that contained responses, this difference was the same for both High- and Low-creative groups. Thus, it seems unlikely that fast response times affected group classification results. Additional analyses to assess the general influence of artifacts, including on condition classification, showed a low rate of artifacts in the 1-2.5 second window (4.4\%) for all trials, and no difference between conditions. Altogether, these additional findings seem to indicate that response-related activity likely had a negligible effect on classification accuracy. This line of thinking is perhaps most heavily bolstered by the finding that changing the epoch window to $0.5-1.75$, which ends 0.24 seconds before mean RT, actually improved accuracy, in the case of between-group classification. If response-related features had been the driver of this classification, then including this period would have presumably resulted in superior model performance. That being said, it is interesting that when the analysis window was changed from $1-2.5 \mathrm{~s}$ to $0.5-1.75 \mathrm{~s}$, condition classification accuracy decreased. One possible reason for changes in the opposite direction for condition classification vs. creativity-based classification is that an earlier window 
somehow favors creativity-based classification, while a later window somehow favors condition classification. One possible explanation for this is that individual differences that are present before creative ideation begins in earnest can be discerned algorithmically. In contrast, condition-based differences are more readily discerned after ideation is well underway. There is also still the matter of condition differences based on response-related, late-window features, but the likelihood that such features affected condition classification results in a meaningful way in this study seems low.

\section{Future Directions}

Future research could employ a timed ideation period for both AUT conditions, thereby avoiding response-related EEG data. The period just prior to response could also be a focus of analysis, given the changes that occur toward the end of creative ideation (Rominger et al., 2019; Schwab et al., 2014). Fast responses in the Normal condition in the present study left a very narrow window between stimulus onset and task response. This meant that many responselocked epochs of interest would contain both stimulus onset and the pre-stimulus period. All things considered, a longer "forced thinking" period, as used by Schwab et al. (2014) would be of benefit in future studies.

Further use of ML in creativity research has the potential to identify classification features that have previously been overlooked, potentially stimulating new lines of research. Future EEG/ML creativity studies could include measures such as functional connectivity, eventrelated potentials, and others. Real-time ML-based neurofeedback is also an enticing option. This study is a step in these directions. 


\section{Conclusions}

In conclusion, the potential value of ML in creativity research is a virtually untapped resource, and the applications could extend well outside of EEG-focused studies. For example, future studies could examine if natural language processing can be used to perform consistent, objective assessments of written or verbal creative task responses, or if image processing ML could do the same for drawings. Certainly, there are other physiological measures to which ML could be applied. Indeed, ML has been used to make predictions based on fMRI data, and even gene-brain interactions, both of which are relevant to investigations of creativity (Ren, Yang, \& Qiu, 2019). Considering the growing ubiquity of ML and its successful implementation in numerous branches of scientific research, it is likely that the field of creativity research would be well-served by a broader adoption of its use. 


\section{References}

Abiri, R., Borhani, S., Sellers, E. W., Jiang, Y., \& Zhao, X. (2018). A comprehensive review of EEG-based brain-computer interface paradigms. Journal of Neural Engineering, 16(1), 011001.

Agnoli, S., Zanon, M., Mastria, S., Avenanti, A., \& Corazza, G. E. (2019). Predicting response originality through brain activity: An analysis of changes in EEG alpha power during the generation of alternative ideas. NeuroImage, 116385.

Avinash, T., Dikshant, L., \& Seema, S. (2018). Methods of neuromarketing and implication of the frontal theta asymmetry induced due to musical stimulus as choice modeling. Procedia Computer Science, 132, 55-67.

Beaty, R. E., Seli, P., \& Schacter, D. L. (2019). Network neuroscience of creative cognition: mapping cognitive mechanisms and individual differences in the creative brain. Current opinion in behavioral sciences, 27, 22-30.

Bell, A. J., \& Sejnowski, T. J. (1995). An information-maximization approach to blind separation and blind deconvolution. Neural computation, 7(6), 1129-1159.

Benedek, M. (2018). The neuroscience of creative idea generation. In Exploring Transdisciplinarity in Art and Sciences (pp. 31-48). Springer, Cham.

Benedek, M., Bergner, S., Könen, T., Fink, A., \& Neubauer, A. C. (2011). EEG alpha synchronization is related to top-down processing in convergent and divergent thinking. Neuropsychologia, 49(12), 3505-3511.

Benedek, M., Schickel, R. J., Jauk, E., Fink, A., \& Neubauer, A. C. (2014). Alpha power increases in right parietal cortex reflects focused internal attention. Neuropsychologia, 56, 393-400. 
Bhattacharyya, S., Khasnobish, A., Konar, A., Tibarewala, D. N., \& Nagar, A. K. (2011, April). Performance analysis of left/right hand movement classification from EEG signal by intelligent algorithms. In 2011 IEEE Symposium on Computational Intelligence, Cognitive Algorithms, Mind, and Brain (CCMB) (pp. 1-8). IEEE.

Boostani, R., Karimzadeh, F., \& Nami, M. (2017). A comparative review on sleep stage classification methods in patients and healthy individuals. Computer Methods and Programs in Biomedicine, 140, 77-91.

Boot, N., Baas, M., Mühlfeld, E., de Dreu, C. K., \& van Gaal, S. (2017). Widespread neural oscillations in the delta band dissociate rule convergence from rule divergence during creative idea generation. Neuropsychologia, 104, 8-17.

Camarda, A., Salvia, E., Vidal, J., Weil, B., Poirel, N., Houde, O., Borst, G., \& Cassotti, M. (2018). Neural basis of functional fixedness during creative idea generation: an EEG study. Neuropsychologia, 118, 4-12.

Carroll, E. A., \& Latulipe, C. (2012, May). Triangulating the personal creative experience: selfreport, external judgments, and physiology. In Proceedings of Graphics Interface 2012(pp. 53-60). Canadian Information Processing Society.

Cavanagh, J. F., \& Frank, M. J. (2014). Frontal theta as a mechanism for cognitive control. Trends in Cognitive Sciences, 18(8), 414-421.

Christensen, L. R., \& Abdullah, M. A. (2018, May). EEG emotion detection review. In 2018 IEEE Conference on Computational Intelligence in Bioinformatics and Computational Biology (CIBCB) (pp. 1-7). IEEE.

Clayton, M. S., Yeung, N., \& Kadosh, R. C. (2015). The roles of cortical oscillations in sustained attention. Trends in Cognitive Sciences, 19(4), 188-195. 
Delorme, A., \& Makeig, S. (2004) EEGLAB: an open source toolbox for analysis of single-trial EEG dynamics. Journal of Neuroscience Methods, 134(1), 9-21.

Duan, R. N., Wang, X. W., \& Lu, B. L. (2012, November). EEG-based emotion recognition in listening music by using support vector machine and linear dynamic system. In International Conference on Neural Information Processing (pp. 468-475). Springer, Berlin, Heidelberg.

Fink, A., \& Benedek, M. (2014). EEG alpha power and creative ideation. Neuroscience \& Biobehavioral Reviews, 44, 111-123.

Fink, A., \& Neubauer, A. C. (2008). Eysenck meets Martindale: The relationship between extraversion and originality from the neuroscientific perspective. Personality and Individual Differences, 44(1), 299-310.

Gabard-Durnam, L. J., Mendez Leal, A. S., Wilkinson, C. L., \& Levin, A. R. (2018). The Harvard Automated Processing Pipeline for Electroencephalography (HAPPE): standardized processing software for developmental and high-artifact data. Frontiers in neuroscience, $12,97$.

Giannakaki, K., Giannakakis, G., Farmaki, C., \& Sakkalis, V. (2017, June). Emotional state recognition using advanced machine learning techniques on EEG data. In 2017 IEEE 30th International Symposium on Computer-Based Medical Systems (CBMS) (pp. 337342). IEEE.

Guilford, J. P. (1967). Creativity: Yesterday, today and tomorrow. The Journal of Creative Behavior, 1(1), 3-14. 
Hadjidimitriou, S. K., \& Hadjileontiadis, L. J. (2012). Toward an EEG-based recognition of music liking using time-frequency analysis. IEEE Transactions on Biomedical Engineering, 59(12), 3498-3510.

Jauk, E., Benedek, M., \& Neubauer, A. C. (2012). Tackling creativity at its roots: Evidence for different patterns of EEG alpha activity related to convergent and divergent modes of task processing. International Journal of Psychophysiology, 84(2), 219-225.

Jawed, S., Amin, H. U. U., Malik, A. S., \& Faye, I. (2019). Classification of visual and nonvisual learners using Electroencephalographic alpha and gamma activities. Frontiers in Behavioral Neuroscience, 13, 86.

Kothe, C. A., \& Makeig, S. (2013). BCILAB: a platform for brain-computer interface development. Journal of neural engineering, 10(5), 056014.

Lin, Y. P., Wang, C. H., Jung, T. P., Wu, T. L., Jeng, S. K., Duann, J. R., \& Chen, J. H. (2010). EEG-based emotion recognition in music listening. IEEE Transactions on Biomedical Engineering, 57(7), 1798-1806.

Martindale, C., \& Hasenfus, N. (1978). EEG differences as a function of creativity, stage of the creative process, and effort to be original. Biological Psychology, 6(3), 157-167.

Martindale, C., \& Mines, D. (1975). Creativity and cortical activation during creative, intellectual and EEG feedback tasks. Biological Psychology, 3(2), 91-100.

Orru, G., Pettersson-Yeo, W., Marquand, A. F., Sartori, G., \& Mechelli, A. (2012). Using support vector machine to identify imaging biomarkers of neurological and psychiatric disease: a critical review. Neuroscience \& Biobehavioral Reviews, 36(4), 1140-1152. 
Putze, F., Scherer, M., \& Schultz, T. (2016, October). Starring into the void? Classifying Internal vs. External Attention from EEG. In Proceedings of the 9th Nordic Conference on Human-Computer Interaction (pp. 1-4).

Ramoser, H., Muller-Gerking, J., \& Pfurtscheller, G. (2000). Optimal spatial filtering of single trial EEG during imagined hand movement. IEEE transactions on rehabilitation engineering, 8(4), 441-446.

Ren, Z., Yang, W., \& Qiu, J. (2019). Neural and genetic mechanisms of creative potential. Current Opinion in Behavioral Sciences, 27, 40-46.

Ritter, S. M., Abbing, J., \& Van Schie, H. T. (2018). Eye-closure enhances creative performance on divergent and convergent creativity tasks. Frontiers in Psychology, 9, 1315.

Rominger, C., Papousek, I., Perchtold, C. M., Benedek, M., Weiss, E. M., Schwerdtfeger, A., \& Fink, A. (2019). Creativity is associated with a characteristic U-shaped function of alpha power changes accompanied by an early increase in functional coupling. Cognitive, Affective, \& Behavioral Neuroscience, 19(4), 1012-1021.

Rossi, F., \& Villa, N. (2006). Support vector machine for functional data classification. Neurocomputing, 69(7-9), 730-742.

Schwab, D., Benedek, M., Papousek, I., Weiss, E. M., \& Fink, A. (2014). The time-course of EEG alpha power changes in creative ideation. Frontiers in Human Neuroscience, 8, 310.

Sternberg, R. J., \& Lubart, T. I. (1996). Investing in creativity. American psychologist, 51(7), 677.

Stevens Jr, C. E., \& Zabelina, D. L. (2019). Creativity comes in waves: an EEG-focused exploration of the creative brain. Current Opinion in Behavioral Sciences, 27, 154-162. 
Van Putten, M. J., Olbrich, S., \& Arns, M. (2018). Predicting sex from brain rhythms with deep learning. Scientific reports, 8(1), 1-7.

Winkler, I., Brandl, S., Horn, F., Waldburger, E., Allefeld, C., \& Tangermann, M. (2014).

Robust artifactual independent component classification for BCI practitioners. Journal of neural engineering, 11(3), 035013.

Wokke, M. E., Ridderinkhof, K. R., \& Padding, L. (2018). Creative minds are out of control: Mid frontal theta and creative thinking. bioRxiv, 370494.

Zhou, S., Chen, S., Wang, S., Zhao, Q., Zhou, Z., \& Lu, C. (2018). Temporal and spatial patterns of neural activity associated with information selection in open-ended creativity. Neuroscience, 371, 268-276. 


\section{Appendix}

List of objects used in the present study in the Alternate Uses Task (AUT)

1. Brick

2. Plate

3. Rope

4. Toothbrush

5. Broom

6. Fork

7. Flowerpot

8. Tire

9. Shovel

10. Coffee Cup

11. Chair

12. Book

13. Doormat

14. Ruler

15. Belt

16. Garbage Can

17. Rake

18. Curtain

19. Spoon

20. Comb

21. Garden Hose

22. Pillow

23. Umbrella

24. Boots

25. Magazine

26. Dustpan

27. Purse

28. Hammer

29. Pencil

30. Scissors 